\title{
Cooperative Multipath-assisted Navigation and Tracking: A Low-Complexity Approach
}

\author{
Josef Kulmer, Erik Leitinger, Paul Meissner, and Klaus Witrisal \\ Graz University of Technology, Signal Processing and Speech Communications, \\ Inffeldgasse 16c, 8010 Graz, Austria \\ \{kulmer, erik.leitinger, paul.meissner, witrisal\}@tugraz.at
}

\begin{abstract}
Cooperative agent networks are a common technology for localization nowadays, nevertheless it is still illusive to reach a certain level of accuracy and robustness for wireless applications, especially in indoor environments which are characterized by harsh multipath condition. In this work we introduce a cooperative low complexity algorithm that utilizes multipath components for localization that are seen as impairment in other respects. The algorithm uses two types of measurements: (i) bistatic measurements between agents and (ii) monostatic (bat-like) measurements by the individual agents.

Simulations that use data generated from a realistic channel model, show the applicability of the methodology and the high level of accuracy that can be reached.
\end{abstract}

\section{Introduction}

Location awareness is a key component of many future wireless applications. Achieving the needed level of accuracy is still elusive, especially in indoor environments which are characterized by harsh multipath conditions. Promising candidate systems thus either use sensing technologies that provide remedies against multipath or they fuse information from multiple information sources [1, 2].

In Multipath-assisted indoor navigation and tracking (MINT) [3, 4] multipath components (MPCs) can be associated to the local geometry using a known floor plan. In this way, MPCs can be seen as signals from additional (virtual) anchors (VAs). Ultra-wideband (UWB) signals are used because of their superior time resolution and to facilitate the separation of MPCs. Hence, additional position-related information is exploited that is contained in the radio signals. All other - not geometrically modelled - propagation effects included in the signals constitute interference to the useful position-related information and are called diffuse multipath (DM) [5]. Insight on the position-related information that is conveyed in the signals can be gained by an analysis of performance bounds, such as the Cramér-Rao lower bound (CRLB) 4]. In [4, the CRLB for cooperative MINT was derived using bistatic measurements between agents and monostatic measurements from an agent itself. The same measurement model will be used in this work. 


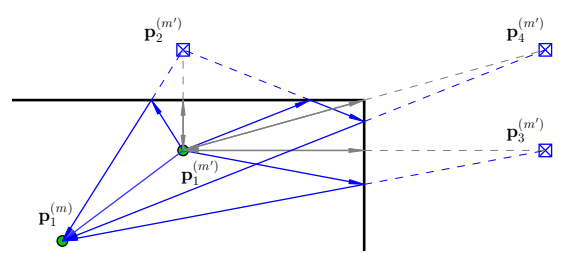

Fig. 1. Illustration of multipath geometry using VAs for (i) bistatic transmissions (blue) between an agents and for (ii) a monostatic measurement (gray) by an agent.

Cooperation between agents is another method to increase the amount of available information [11] and thus to reduce the localization outage. Based on a message passing methodology and particle filtering agents were tracked using bistatic measurements between agents and monostatic measurements from the agents itself.

In this work, we present a low-complexity variant of 7 that is based on dataassociation (DA) and extended Kalman filtering (EKF) [9]. The method relies on the same factor graph as presented in 7, but in contrast it just uses the extracted MPC delays and complex path amplitude 1 instead of the complete received signals. The key contributions of this paper are (i) incorporate VAs into a joint state space with the agents, and (ii) formulate the cooperative algorithm that uses DA of MPC delays with according VAs and an EKF for tracking the joint state of the agents and the according VAs.

\section{Problem Formulation}

We assume $M$ agents at positions $\mathbf{p}_{1}^{(m)}$ with $m \in \mathcal{N}_{m}=\{1,2, \ldots, M\}$, which cooperate with one another. As outlined in the introduction, every agent conducts a monostatic measurement, meaning it emits a pulse and receives the multipath signal reflected by the environment, and conventional bistatic measurements with all other agents and the fixed anchors. All bistatic and monostatic measurements are distributed such that every agent is able to exploit information from any of its received and/or transmitted signals.

Fig. 11illustrates the geometric model for multipath-assisted positioning. A signal exchanged between the agents $m^{\prime}$ and $m$ at positions $\mathbf{p}_{1}^{\left(m^{\prime}\right)}$ and $\mathbf{p}_{1}^{(m)}$, respectively, contains specular reflections at the room walls, indicated by the black lines. These reflections can be modeled geometrically using VAs $\mathbf{p}_{k}^{\left(m^{\prime}\right)}$, mirror images of the anchor w.r.t. walls that can be computed from the floor plan [3. We call this the bistatic setup. If the agents are equipped accordingly, they can use monostatic measurements, indicated by the gray lines. Here, the node at $\mathbf{p}_{k}^{\left(m^{\prime}\right)}$ acts as anchor for itself with its own set of VAs.

\footnotetext{
${ }^{1}$ These are used to compute online, the reliability measure of the MPCs in form a signal-to-interference-plus-noise-ration (SINR) that is used to compute the according MPC's delay uncertainty.
} 


\subsection{Signal Model}

In this Section, we simplify the setup - for the ease of readability - to a single (fixed) anchor located at position $\mathbf{p}_{1} \in \mathbb{R}^{2}$ and one agent at position $\mathbf{p} \in \mathbb{R}^{2}$. Note that two-dimensional position coordinates are used throughout the paper, for the sake of simplicity. A baseband UWB signal $s(t)$ is exchanged between the anchor and the agent. The corresponding received signal is modeled as 4 ]

$$
r(t)=\sum_{k=1}^{K} \alpha_{k} s\left(t-\tau_{k}\right)+(s * \nu)(t)+w(t)
$$

where $\left\{\alpha_{k}\right\}$ and $\left\{\tau_{k}\right\}$ are the complex amplitudes and delays of the deterministic MPCs, respectively. We model these delays by VAs at positions $\mathbf{p}_{k} \in \mathbb{R}^{2}$, yielding $\tau_{k}=\frac{1}{c}\left\|\mathbf{p}-\mathbf{p}_{k}\right\|=\frac{1}{c} d\left(\mathbf{p}_{k}, \mathbf{p}\right)$, with $k=1 \ldots K$, where $c$ is the speed of light and $d(\cdot)$ is the Euclidean distance. $K$ is equivalent to the number of visible VAs at the agent position p. We assume the energy of $s(t)$ is normalized to one.

The DM $(s * \nu)(t)$ is modeled as a zero-mean Gaussian random process which is non-stationary in the delay domain and colored due to the spectrum of $s(t)$. For DM we assume uncorrelated scattering along the delay axis $\tau$, hence the autocorrelation function (ACF) of $\nu(t)$ is given by $K_{\nu}(\tau, u)=\mathbb{E}_{\nu}\left\{\nu(\tau)[\nu(u)]^{*}\right\}=$ $S_{\nu}(\tau) \delta(\tau-u)$, where $S_{\nu}(\tau)$ is the PDP of DM at the agent position $\mathbf{p}$. The DM process is assumed to be quasi-stationary in the spatial domain, which means that $S_{\nu}(\tau)$ does not change in the vicinity of position $\mathbf{p}$ 4. Finally, $w(t)$ denotes an additive white Gaussian noise (AWGN) process with double-sided power spectral density (PSD) of $N_{0} / 2$.

\subsection{Channel Estimation}

The arrival time estimation $\hat{\tau}_{k, n}^{\left(m, m^{\prime}\right)}$ at time step $n$ between two agents at positions $\mathbf{p}_{1, n}^{(m)}$ and $\mathbf{p}_{1, n}^{\left(m^{\prime}\right)}$, where $m, m^{\prime} \in \mathcal{N}_{m}$, is realized as an iterative least-squares approximation of the received signal [9]. The according path amplitudes $\hat{\alpha}_{k, n}^{\left(m, m^{\prime}\right)}$ are estimated using a projection of the received signal $r_{n}^{\left(m, m^{\prime}\right)}(t)$ onto a unitenergy pulse $s\left(t-\hat{\tau}_{k, n}^{\left(m, m^{\prime}\right)}\right)$. The number of estimated MPCs $\hat{K}_{n}^{\left(m, m^{\prime}\right)}$ should be chosen according to the number of expected specular paths in an environment. The finite set of measured delays is written as $\mathcal{Z}_{n}^{(m)}=\bigcup_{m^{\prime}} \mathcal{Z}_{n}^{\left(m, m^{\prime}\right)}=$ $\bigcup_{m^{\prime}}\left\{\hat{d}_{k, n}^{\left(m, m^{\prime}\right)}\right\}_{k=1}^{\hat{K}_{n}^{\left(m, m^{\prime}\right)}}$, where $\hat{d}_{k, n}^{\left(m, m^{\prime}\right)}=c \hat{\tau}_{k, n}^{\left(m, m^{\prime}\right)}$.

\subsection{Data Association (DA)}

The set of expected MPC delays $\mathcal{D}_{n}^{\left(m, m^{\prime}\right)}=\left\{d\left(\mathbf{p}_{n, k}^{\left(m^{\prime}\right)}, \mathbf{p}_{1, n}^{(m)}\right): \mathbf{p}_{n, k}^{\left(m, m^{\prime}\right)} \in \mathcal{A}_{n}^{\left(m, m^{\prime}\right)}\right\}$ is computed from the distances of each VA in $\mathcal{A}_{n}^{\left(m, m^{\prime}\right)}$ to the predicted position $\mathbf{p}_{1, n}^{(m)}$ of agent $m$ at time step $n$. As $\mathcal{D}_{n}^{\left(m, m^{\prime}\right)}$ and the set of measured delays $\mathcal{Z}_{n}^{\left(m, m^{\prime}\right)}$ are sets of usually different cardinalities, i.e. $\left|\mathcal{Z}_{n}^{\left(m, m^{\prime}\right)}\right|=\hat{K}_{n}^{\left(m, m^{\prime}\right)} \neq$ 
$\left|\mathcal{D}_{n}^{\left(m, m^{\prime}\right)}\right|=K_{n}^{\left(m, m^{\prime}\right)}$, no conventional distance measure is defined and therefore there is no straightforward way of an association. We employ a well-known multitarget miss-distance, the optimal sub-pattern assignment (OSPA) metric [10]. As described in [9, 6, after the DA was applied for all agents, the following union sets are defined: (i) the set of associated discovered (and optionally a-priori known) VAs $\mathcal{A}_{n, \text { ass }}^{(m)}=\bigcup_{m^{\prime}} \mathcal{A}_{n, \text { ass }}^{\left(m, m^{\prime}\right)}$, (ii) the corresponding set of associated measurements $\mathcal{Z}_{\text {timestepsym }, \text { ass }}^{(m)}=\bigcup_{m^{\prime}} \mathcal{Z}_{n, \text { ass }}^{\left(m, m^{\prime}\right)}$.

\section{State Space and Measurement Model}

The state dynamics are characterized by a linear, constant-velocity motion model. Each agent $\mathbf{x}_{n}^{(m)}$ is described by its position $\mathbf{p}_{1, n}^{(m)}$ and velocity $\mathbf{v}_{1, n}^{(m)}$ according to $\mathbf{x}_{n}^{(m)}=\left[\left(\mathbf{p}_{1, n}{ }^{(m)}\right)^{\mathrm{T}},\left(\mathbf{v}_{1, n}{ }^{(m)}\right)^{\mathrm{T}}\right]^{\mathrm{T}}$. The position of the agent is mirrored at a each wall segment in order to obtain the positions of the corresponding VAs $\mathbf{p}_{k, n}^{(m)}$. The orientation of the wall segments determine the relation between the movement gradients of the agent and the corresponding VAs. We describe this relation by introducing a VA transition matrix $\mathbf{P}_{k}^{(m)}$ (cf. [4]). The state space model for agent $m$ is thus characterized by

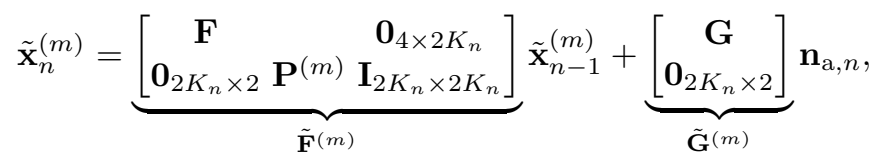

with

$$
\mathbf{F}=\left[\begin{array}{cccc}
1 & 0 & \Delta T & 0 \\
0 & 1 & 0 & \Delta T \\
0 & 0 & 1 & 0 \\
0 & 0 & 0 & 1
\end{array}\right], \quad \mathbf{G}=\left[\begin{array}{cc}
\frac{\Delta T^{2}}{2} & 0 \\
0 & \frac{\Delta T^{2}}{2} \\
\Delta T & 0 \\
0 & \Delta T
\end{array}\right]
$$

$\tilde{\mathbf{x}}_{n}^{(m)}=\left[\mathbf{x}_{n}^{\mathrm{T}}{ }_{n}^{(m)}, \mathbf{p}_{2, n}^{\mathrm{T}_{2, n}^{(m)}}, \ldots \mathbf{p}_{K, n}^{\mathrm{T}}{ }_{K, n}^{(m)}\right]^{\mathrm{T}}$ and $\mathbf{P}^{(m)}=\left[\mathbf{P}_{2}^{\mathrm{T}(m)}, \ldots, \mathbf{P}_{K_{n}}^{\mathrm{T}(m)}\right]^{\mathrm{T}}$ with dimensions $\left(2 K_{n} \times 2\right)$. Under the assumption of independent movement of the agents, the motion model finally results in

$$
\underbrace{\left[\begin{array}{c}
\tilde{\mathbf{x}}_{n}^{(1)} \\
\vdots \\
\tilde{\mathbf{x}}_{n}^{(M)}
\end{array}\right]}_{\hat{\mathbf{x}}_{n}}=\left[\begin{array}{ccc}
\tilde{\mathbf{F}}^{(1)} & & \mathbf{0} \\
& \ddots & \\
\mathbf{0} & & \tilde{\mathbf{F}}^{(M)}
\end{array}\right] \underbrace{\left[\begin{array}{c}
\tilde{\mathbf{x}}_{n-1}^{(1)} \\
\vdots \\
\tilde{\mathbf{x}}_{n-1}^{(M)}
\end{array}\right]}_{\tilde{\mathbf{x}}_{n-1}}+\left[\begin{array}{c}
\tilde{\mathbf{G}}_{n}^{(1)} \\
\tilde{\mathbf{G}}_{n}^{(M)}
\end{array}\right] \mathbf{n}_{\mathrm{a}, n} .
$$

The according linearized measurement model is defined as

$$
\left[\begin{array}{c}
\tilde{\mathbf{z}}_{n}^{(1)} \\
\vdots \\
\tilde{\mathbf{z}}_{n}^{(M)}
\end{array}\right]=\left[\begin{array}{ccc}
\tilde{\mathbf{H}}_{n}^{(1)} & & \mathbf{0} \\
& \ddots & \\
& & \tilde{\mathbf{H}}_{n}^{(M)}
\end{array}\right]\left[\begin{array}{c}
\tilde{\mathbf{x}}_{n}^{(1)} \\
\vdots \\
\tilde{\mathbf{x}}_{n}^{(M)}
\end{array}\right]+\tilde{\mathbf{n}}_{z, n}
$$


where $\tilde{\mathbf{z}}_{n}^{(m)}$ stacks the monostatic measurements from the $m$-th agent and the bistatic measurements to all other agents. The stack vector $\tilde{\mathbf{n}}_{z, n}$ contains the according measurement noise with covariance matrix $\mathbf{R}_{n}$ described in 9. The linearized column-wise stacked measurement matrices $\tilde{\mathbf{H}}_{n}^{(m)}=$ $\left[\left(\tilde{\mathbf{H}}_{n}^{(\eta=1, m)}\right)^{\mathrm{T}}, \ldots,\left(\tilde{\mathbf{H}}_{n}^{(\eta=M, m)}\right)^{\mathrm{T}}\right]^{\mathrm{T}}$ described in (5), with $m, \eta \in \mathcal{N}_{m}$ and $m \neq \eta$. The matrix $\mathbf{H}_{\xi, \mu, n}^{(\eta, \eta, m)}=\left[\frac{\partial d\left(\mathbf{p}_{\mu, n}^{(\eta)}, \mathbf{p}_{1, n}^{(m)}\right)}{\partial x_{\xi, n}^{(\eta)}}, \frac{\partial d\left(\mathbf{p}_{\mu, n}^{(\eta)}, \mathbf{p}_{1, n}^{(m)}\right)}{\partial y_{\xi, n}^{(\eta)}}\right]$ defines the derivatives of the measurement equation w.r.t. the $x$ - and $y$-position coordinates. The upper-left sub-block of (5) holds the derivatives of the monostatic measurement equations w.r.t. the $m$-th agent position and upper diagonal sub-block the according derivatives w.r.t. to the monostatic VA positions of the $m$-th agent. The lowerleft sub-block holds derivatives of the bistatic measurement equations to all other agent positions and according VA positions $(\eta=1 \ldots M$ and $m \neq \eta)$ w.r.t. the $m$-th agent position. The lower-right diagonal sub-block holds the equivalent derivatives w.r.t. to the according bistatic VA positions.

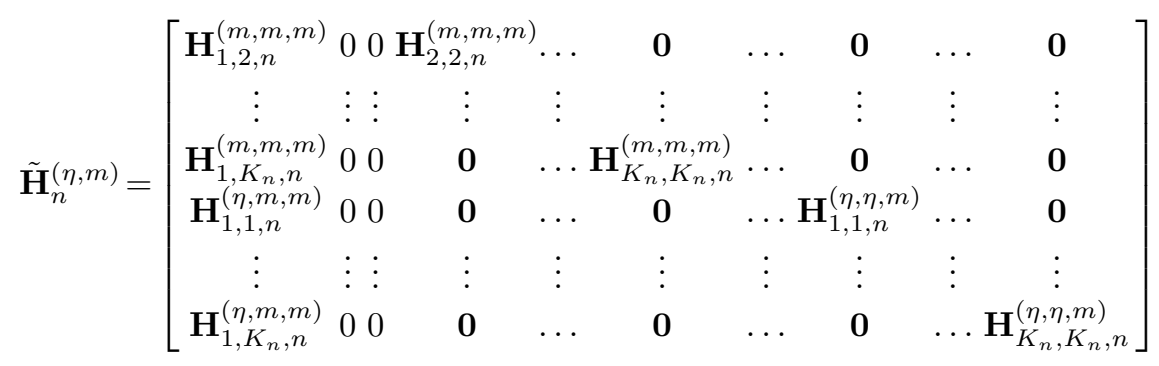

\section{Results}

We evaluate the performance of the proposed algorithm in terms of localization error and computational time using synthetic data in a two-dimensional space. The transmit signal consists of a raised-cosine pulse with a roll-off factor of $R=0.6$, a pulse duration of $T_{\mathrm{p}}=0.5 \mathrm{~ns}$ and unit energy. The received signals of the monostatic and bistatic measurements are modeled according to (11). Each reflection attenuates the pulse by $3 \mathrm{~dB}$. The free-space loss is modeled according to Friis' transmission equation. The parameters of the DM are set according to [4] and the power of the additive white noise is set to $N_{0}=2 \cdot 10^{-16} \mathrm{~W} / \mathrm{Hz}$. In order to achieve a fair comparison to the proposed method in [7] we choose the same parameter setup and simulation scenario as shown in Figure 2. Three agents $m$ move independenly along trajectories under partly non-line-of-sight conditions where we assume a given start position. Figure 2 shows an example of the estimated agent positions $\hat{\mathbf{p}}_{1, n}, \hat{\mathbf{p}}_{2, n}$ and $\hat{\mathbf{p}}_{3, n}$ using the proposed EKFbased algorithm are indicated for every 5 -th position. At each time step $n$ the agents run monostatic and bistatic measurements to the neighboring agents. The utilized likelihood function of [7, eq. (8)] simplifies the proposed system model (Sec. III in [7). We accounted this by changing the likeihood function to eq. 
(7) of [7. Further, 7] undermines the uncertainty of the neighboring beliefs by reducing the size of the neighboring particles to the mean value (see Sec. V-B of [7). We omit this simplification.

The maximum number of extracted MPCs is limited to $K_{n}^{(., .)}=12, \forall n$ (see 2.2). The initial position of each VA $\mathbf{p}_{k, n}^{(m)}$ as well as the corresponding VA transition matrix $\mathbf{P}_{k}^{(m)}$ are calculated in advance. Figure 3 illustrates the cumulative distribution function $(\mathrm{CDF})$ of the localization error of the proposed algorithm (CoMINT EKF) compared to 7] of ten trajectory realizations - each evaluated with 50 monte carlo runs. The comparison reveals the strong influence on performance of localization error and computational demand [7] regarding its implementation scheme of message passing (i.e. particle or parametric message represenation and scheduling). Choosing a sample-based message representation the localization error reduces with increasing number of particles on the cost of computational complexity. Denoting $N$ as the number of particles representing the message passing scheme [7, faces a complexitiy of $\mathcal{O}\left(M N^{2}\right)$ [ 8 . The proposed method has a complexity of $\mathcal{O}\left(M^{2} K_{n}^{3}\right)$ determined by the data association stage 10. Since the number of particles $N$ is much higher compared to the number of extracted MPC $K_{n}^{(., .)}$the proposed method outperforms [7] in terms of computational complexity. We proof this claim by comparison of the average computational time for localization scaled to the proposed method. Depending on the number of particles the average computational time of the proposed method speeds up by a factor of approximately 217, 756 and 2355 for 100, 250 and 500 particles, respectively.

The gain in terms of computational time is established by the assumption of Gaussian distance errors. Figure 3 indicates the influence of this assumption by comparison to [7] with different number of particles of 100, 250 and 500. The proposed method reaches a performance compareable to [7] with a number of particles from $100-250$ where $90 \%$ of the errors are located within $2 \mathrm{~cm}$.

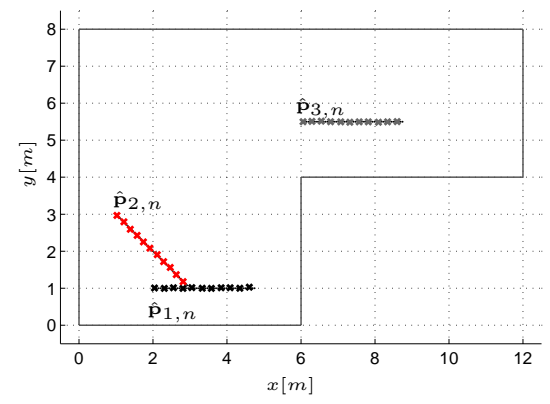

Fig. 2. Simulation scenario as in 7 with three agents moving along different trajectories.

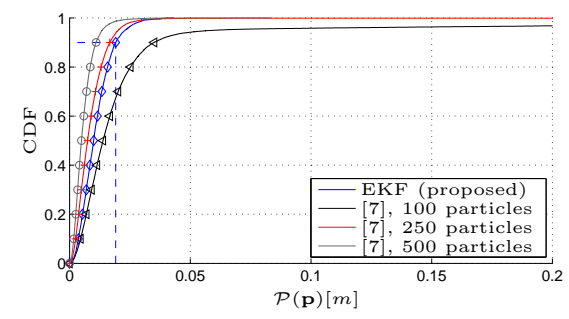

Fig. 3. CDF of the localization error of the proposed algorithm (CoMINT EKF) (blue) compared to 7] with a different size of 500 (gray), 250 (red) and 100 (black) particles of each agent. 


\section{Conclusions}

We have presented an new low-complexity algorithm for cooperative localization of agents using multipath information. The simulation results show that bistatic as well as monostatic measurements contribute a significant amount of information for localizing the agents with a high level of accuracy. The simulation results pinpoint also the robustness, i.e. low level of localization outages, of the cooperative algorithm when multipath information is used from both types of measurements. The most important attained fact it that the proposed lowcomplexity algorithm reaches almost the same performance using several orders of magnitude less computational resources than the particle-based method.

\section{References}

1. Shen, Y., Mazuelas, S., Win, M.: Network Navigation: Theory and Interpretation. IEEE Journal on Selected Areas in Communications (2012)

2. Conti, A., Dardari, D., Guerra, M., Mucchi, L., Win, M.: Experimental Characterization of Diversity Navigation. IEEE Systems Journal (2014)

3. Meissner, P.: Multipath-Assisted Indoor Positioning. Ph.D. thesis, Graz University of Technology (2014)

4. Leitinger, E., Meissner, P., Ruedisser, C., Dumphart, G., Witrisal, K.: Evaluation of Position-related Information in Multipath Components for Indoor Positioning. Selected Areas in Communications, IEEE Journal on (2015)

5. Michelusi, N., Mitra, U., Molisch, A., Zorzi, M.: UWB Sparse/Diffuse Channels, Part I: Channel Models and Bayesian Estimators. IEEE Transactions on Signal Processing (2012)

6. Leitinger, E., Meissner, P., Lafer, M., Witrisal, K.: Simultaneous Localization and Mapping using Multipath Channel Information. In: Communications Workshops (ICC), 2015 IEEE International Conference on (2015)

7. Froehle, M., Leitinger, E., Meissner, P., Witrisal, K.: Cooperative MultipathAssisted Indoor Navigation and Tracking (Co-MINT) Using UWB Signals. In: IEEE ICC Workshop on Advances in Network Localization and Navigation (2013)

8. Lien, J., Ferner, U.J., Srichavengsup, W., Wymeersch, H., Win, M.Z.: A comparison of parametric and sample-based message representation in cooperative localization. International Journal of Navigation and Observation (2012)

9. Meissner, P., Leitinger, E., Witrisal, K.: UWB for Robust Indoor Tracking: Weighting of Multipath Components for Efficient Estimation. IEEE Wireless Communications Letters (2014)

10. Schuhmacher, D., Vo, B.T., Vo, B.N.: A Consistent Metric for Performance Evaluation of Multi-Object Filters. IEEE Transactions on Signal Processing (2008)

11. Wymeersch, H., Lien, J., Win, M.Z.: Cooperative Localization in Wireless Networks. Proceedings of the IEEE (2009) 